VIROLOGY JOURNAL



Influenza A virus induced bacterial otitis media is independent of virus tropism for a2,6-linked sialic acid

Short et al. 


\title{
Influenza A virus induced bacterial otitis media is independent of virus tropism for a2,6-linked sialic acid
}

\author{
Kirsty R Short ${ }^{1 *}$, Marrit N Habets ${ }^{2}$, Jean Payne ${ }^{3}$, Patrick C Reading ${ }^{1,4}$, Dimitri A Diavatopoulos ${ }^{2+}$ \\ and Odilia L Wijburg ${ }^{1+}$
}

\begin{abstract}
Background: Otitis media (OM) affects $\geq 80 \%$ of children before the age of three. OM can arise following co-infection with influenza A virus (IAV) and the bacterium Streptococcus pneumoniae. We have previously shown that H3 IAV strains (such as Udorn/72) induced a higher rate of bacterial OM than $\mathrm{H} 1$ strains (such as PR8/34). This was associated with more efficient replication of $\mathrm{H} 3$ strains in the middle ear.
\end{abstract}

Findings: Here, we assess if the increased replication of IAV strains such as Udorn/72 in the middle ear is dependent upon the binding of the viral HA to a2,6-linked sialic acid. Using murine and in vitro models, the present study shows that recognition of a2,6-linked sialic acid was not required to facilitate bacterial OM.

Conclusions: Taken together, these data suggest that other features of the HA mediate bacterial OM.

Keywords: Otitis media, Viral-bacterial co-infection, Sialic acid, Influenza virus

\section{Findings}

Introduction

Otitis media (OM) is the most frequently diagnosed illness in children under the age of five and can lead to permanent hearing loss [1]. OM is a polymicrobial disease and can arise following co-infection with influenza A virus (IAV) and the bacterium Streptococcus pneumoniae (the pneumococcus) [1]. We and others have demonstrated that infection with IAV facilitates the development of pneumococcal OM [2-4]. Specifically, we showed that H3 IAV strains, such as Udorn/72 (H3N2), induced a higher rate of pneumococcal $\mathrm{OM}$ in mice compared to $\mathrm{H} 1$ strains, such as PR8/34 (H1N1) [5]. This was related to the ability of the $\mathrm{H} 3$ hemagglutinin (HA) to mediate increased viral replication in the middle ear. $\mathrm{H} 3$ viruses also infected human middle ear epithelial cells (HMEECs) at a higher rate than $\mathrm{H} 1$ strains in vitro [5]. Here, we investigate if the increased replication of $\mathrm{H} 3$ strains in the middle ear is related to the sialic acid preference of the HA.

\footnotetext{
*Correspondence: kirsty_short@yahoo.com

${ }^{\dagger}$ Equal contributors

'Department of Microbiology and Immunology, The University of Melbourne, Melbourne, VIC 3010, Australia

Full list of author information is available at the end of the article
}

The HA of IAV binds to terminal sialic acid residues on cell surface glycoproteins and glycolipids, facilitating virus internalisation [6]. Human influenza viruses typically display a preference for sialic acid residues linked to cell surface glycans by an $\alpha 2-6$ linkage [6]. In humans, $\alpha 2-6$ linkages are most prevalent in the upper respiratory tract, whilst both $\alpha 2-3$ and $\alpha 2-6$ linkages are found in the lower respiratory tract [7]. Interestingly, whilst pre1977 H3N2 strains like Udorn/72 recognise both $\alpha 2,3-$ and $\alpha 2,6$-linked sialic acid, later H3N2 strains have evolved to recognise only $\alpha 2,6$-linked sialic acid $[8,9]$. In contrast to humans, $\alpha 2,3$-linked sialic acid dominates the murine respiratory tract [10]. Accordingly, virus strains adapted for murine infections (such as PR8/34) preferentially recognise $\alpha 2,3$-linked sialic acid [9]. Given that we have previously demonstrated that the Udorn/72 HA mediates efficient viral replication in the middle ear (and thus triggers bacterial OM) whilst the PR8/34 HA does not [5], the recognition of $\alpha 2,6$-linked sialic acid may facilitate increased viral replication in the middle ear. However, the sialic acid linkages present in the middle ears of infant C57BL/6 mice has, to the best of our knowledge, yet to be described. Here, we use a mouse 
model of OM and in vitro cultured HMEECs to show that increased replication of select $\mathrm{H} 3$ influenza virus strains in the middle ear is independent of tropism for a2,6-linked sialic acid.

\section{Materials \& methods}

\section{Viral and bacterial strains}

The bioluminescent $S$. pneumoniae strain EF3030 ${ }^{\text {lux }}$ (type 19F) was used in all experiments [11]. A/Udorn/307/72 (H3N2; Udorn72), A/Port Chalmers/1/73 (H3N2; Port Chalmers/73) and HKx31 (H3N2) were grown in embryonated eggs [4]. Horse-serum resistant $\left(\mathrm{HS}^{\mathrm{R}}\right)$ mutants were selected by growing the parent virus in embryonated eggs in the presence of non-immune horse serum. Tropism of all $\mathrm{HS}^{\mathrm{R}}$ strains for only $\alpha 2,3$-linked sialic acid was confirmed by the desialyation-resialyation of red blood cells [9], and was consistent with previous publications with these strains [9].

\section{Infection of mice}

Animal experiments were approved by the Animal Ethics Committee of The University of Melbourne (APP0811053 \& APP1011872). C57BL/6 mice were bred and housed under specific pathogen-free conditions. Five-day old C57BL/6 mice were colonised intranasally (i.n.) with $2 \times$ $10^{3}$ colony forming units (CFU) of S. pneumoniae EF3030 ${ }^{\text {Lux }}$ or PBS in $3 \mu$ Ls. At 14-days of age, mice were infected i.n. with $20(\mathrm{PR} 8 / 34)$ or $10^{2.5}$ (all other virus strains) plaque forming units (PFU) of IAV in $3 \mu \mathrm{Ls}$. Six days post-IAV infection, organs were collected for analysis.

\section{Enumeration of bacterial and viral load}

Tissues used to quantify bacterial and viral load were collected and processed as described previously [11].

Infection and staining of HMEECs

HMEECs [12] were kindly donated by Prof. David Lim (House Ear Institute, $\mathrm{CA}$ ) and were infected and stained
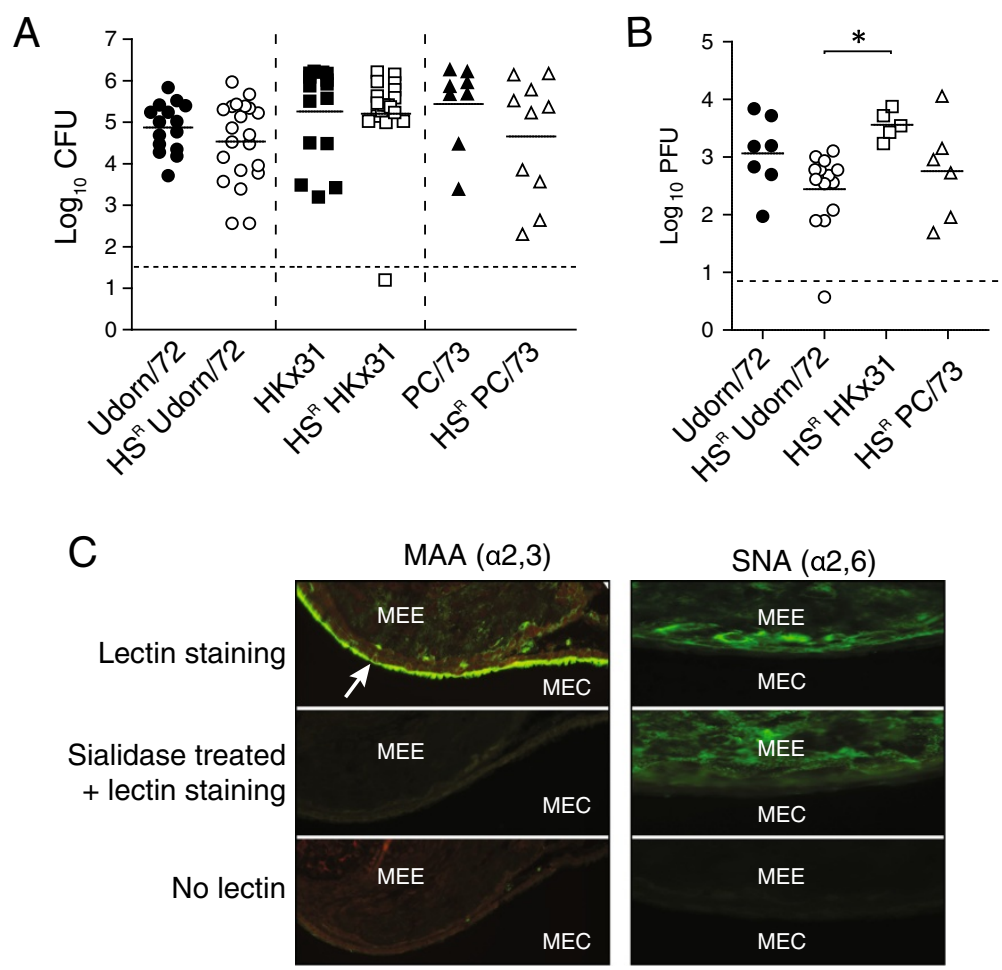

Figure 1 a2,6-linked sialic acid recognition does not affect the development of pneumococcal OM. A. Titres of S. pneumoniae EF3030 in the middle ears of mice six-days after i.n. infection with wild-type or horse-serum resistant $\left(H S^{R}\right)$ IAV strains. Bacterial counts are represented as the average titre derived from the left and right ear of each mouse. Data is pooled from a minimum of two independent experiments. Statistical significance was determined using a Mann-Whitney U-Test between the relevant wild-type or $\mathrm{HS}^{\mathrm{R}}$ strain. Dashed line indicates the detection limit of the assay. B. Titres of IAV in the middle ears of mice six-days after i.n. infection with wild-type or horse-serum resistant (HS $\left.{ }^{R}\right)$ IAV strains. Viral tires are represented as the average titre derived from the left and right ear of each mouse. Data is pooled from a minimum of two independent experiments. Statistical significance was determined using a One-Way ANOVA with a Bonferroni post-correction and is denoted by an asterisk $(p<0.05)$. Dashed line indicates the detection limit of the assay. $\mathbf{C}$. Sialic acid expression in the murine middle ear. Sections were labeled with Maackia amurensis agglutinin (MAA; a2,3-linked sialic acid) or Sambucus nigra agglutinin (SNA; a2,6-linked sialic acid) with or without pre-treatment with a sialidase. Positive staining is shown by an arrow. MEE: Middle Ear Epithelium; MEC: Middle Ear Cavity. 
as described previously [5]. The percentage of infected cells was calculated by counting a minimum of 80 cells per well, where each data point was the average of an experimental duplicate.

\section{Lectin staining}

Middle ears were collected and sectioned for histology essentially as described previously [4]. Lectin staining on middle ear sections was performed using the DIG glycan differentiation kit (Roche Diagnostics, Germany). Briefly, sections were incubated for 1 hour at $37^{\circ} \mathrm{C}$ in PBS or PBS supplemented with $400 \mathrm{mU} / \mathrm{mL}$ Vibrio cholerae sialidase (Sigma, USA). Sections were subsequently labelled with DIG-labelled Maackia amurensis agglutinin (MAA; $\alpha 2,3-$ linked sialic acid) or Sambucus nigra agglutinin (SNA; $\alpha 2,6$-linked sialic acid) and stained with anti-DIG-FITC (Roche, USA). Autofluorescence was quenched using $0.01 \%$ (w/v) Evans blue (Sigma).

HMEECs were incubated with PBS supplemented with $4 \mathrm{mM} \mathrm{CaCl} \pm 500 \mathrm{mU} / \mathrm{mL}$ Vibrio cholerae sialidase (Sigma) for 120 minutes at 37 degrees. Cells were subsequently washed and stained with biotinylated Maackia amurensis Lectin (MAL II) (Vector Laboratories, USA), or with biotinylated Sambucus nigra lectin (SNA I) (EY Laboratories, USA). Cells were once again washed, incubated with streptavidin-conjugated APC (BD Biosciences, USA) and lectin binding was measured on a LSR II flow cytometer (BD Biosciences, USA). Data was analysed with FlowJo version 7.6.5 (TreeStar Inc, Ashland, OR).

\section{Results \& discussion}

\section{OM is not associated with tropism of the viral HA for} a2,6-linked sialic acid

To determine if recognition of $\alpha 2,6$-linked sialic acid was important in the development of secondary bacterial OM, we assessed the ability of a horse serum-resistant mutant $\left(\mathrm{HS}^{\mathrm{R}}\right)$ of Udorn/72 to cause pneumococcal OM. This virus has a single amino acid substitution at residue 226 of the HA that changes its specificity from dual recognition of $\alpha 2,3-$ and $\alpha 2,6$-linked sialic acid, to $\alpha 2,3$-linked sialic acid only [9]. Mice colonised with S. pneumoniae at 5-days old were then infected with wild-type Udorn/72 or $\mathrm{HS}^{\mathrm{R}}$ Udorn/72 at 14-days old. Pneumococcal OM was assessed by measuring bacterial titres in the middle ear six-days post-IAV infection. This time-point was selected as we have previously shown that six days after IAV infection is the peak of bacterial OM in infant mice [4]. Mice infected with $\mathrm{HS}^{\mathrm{R}}$ Udorn/72 displayed no significant difference in middle ear bacterial titres compared to mice infected with wild-type Udorn/72 (Figure 1A; Mann-Whitney $U$-Test, $\mathrm{p}>0.05)$. Accordingly, there was no difference in viral replication between wild-type and $\mathrm{HS}^{\mathrm{R}}$ Udorn/72 in the middle ear six-days post IAV infection (Figure 1B; One-Way ANOVA with a Bonferroni post-correction, $\mathrm{p}>0.05$ ). Like
Udorn/72, the H3N2 viruses $\mathrm{HK} \times 31$ and $\mathrm{PC} / 73$ also replicated efficiently in the middle ear and facilitated bacterial OM [5]. As these viruses also display a dual-receptor specificity $[9,13]$, we used $\mathrm{HS}^{\mathrm{R}}$ mutants to assess if bacterial OM caused by HKx31 and PC/73 was also independent of tropism for $\alpha 2,6$-linked sialic acid. $H S^{\mathrm{R}} H K x 31$ and $H S^{\mathrm{R}}$ $\mathrm{PC} / 73$ did not mediate differential bacterial replication in the middle ear relative to their wild-type parental strains (Figure 1A; Mann-Whitney $U$-Test, $\mathrm{p}>0.05$ ). Similarly, there were no significant differences in the titres of these viruses in the middle ear relative to Udorn/72 (Figure 1B; One-Way ANOVA with a Bonferroni post-correction, $\mathrm{p}>0.05)$ and their replication was equivalent to relevant parental strains [5]. These observations were supported by immunofluorescent staining of middle ears of uninfected mice, which demonstrated that this site is rich in $\alpha 2,3-$ linked sialic acid (Figure 1C). In contrast, only nonspecific background staining was observed for $\alpha 2,6$-linked sialic acid (Figure 1C). The middle ears of S. pneumoniae, IAV and co-infected mice were also rich in $\alpha 2,3$-linked sialic acid (Additional file 1). Furthermore, we have previously demonstrated that secondary pneumococcal $\mathrm{OM}$ is independent of the viral NA and that the pneumococci in the ear localise to the lumen rather than the middle ear

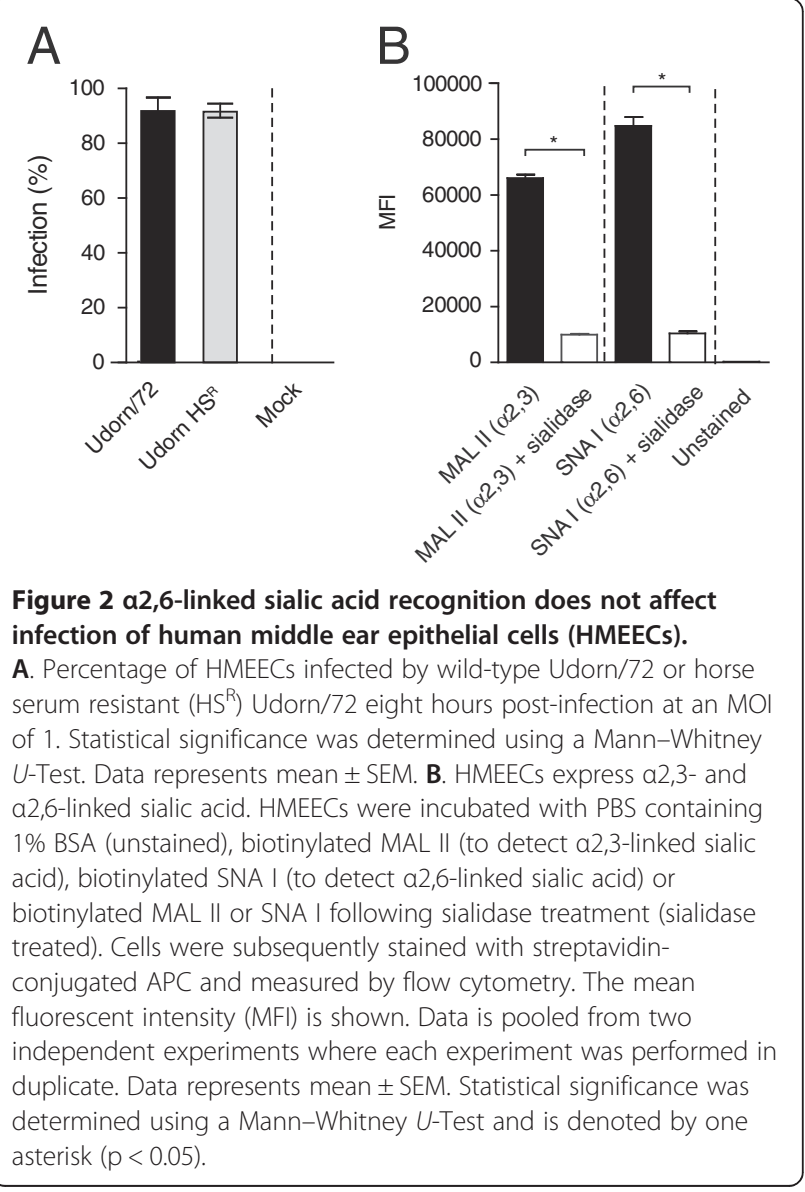


epithelium $[4,5]$. Thus, $\alpha 2,6$-linked sialic acid recognition is not the critical determinant of virus replication in the middle ear or secondary bacterial OM.

\section{Tropism for a2,6-linked sialic acid does not determine the in vitro infection rate of human middle ear epithelial cells by Udorn/72}

To assess the relevance of these findings to human infections, HMEECs were infected with Udorn/72 or $\mathrm{HS}^{\mathrm{R}}$ Udorn/72. $\mathrm{HS}^{\mathrm{R}}$ Udorn/72 was not significantly reduced in its ability to infect HMEECs when compared to the wild-type Udorn/72 (Figure 2A; Mann-Whitney $U$-Test, $p>0.05)$. This suggests that recognition of $\alpha 2,6$-linked sialic acid on HMEECs by the Udorn/72 HA is not the major factor in determining the rate of infection. Consistent with this hypothesis, HMEECs expressed both $\alpha 2,6$ and $\alpha 2,3$-linked sialic acid on the cell surface (Figure $2 B$ ). The specificity of this binding was confirmed by the reduced number of fluorescent cells observed following sialidase treatment of HMEECs (Figure 2B).

In summary, we have previously demonstrated that $\mathrm{H} 3$ viruses (such as Udorn/72) with dual specificity for $\alpha 2,6$ and $\alpha 2,3-$ linked sialic acid facilitate bacterial OM. In contrast, $\mathrm{H} 1$ viruses (such as PR8/34) which only recognise $\alpha 2,3$-linked sialic acid, do not facilitate bacterial OM. We therefore reasoned that recognition of $\alpha 2,6$-linked sialic acid may promote increased viral replication in the middle ear and thus bacterial OM. However, H3 viruses with a specificity for only $\alpha 2,3$-linked sialic acid were still effective at inducing bacterial OM, replicating in the middle ear and infecting HMEECs. At present, it remains unclear what alternative feature of the H3 HA mediates increased viral replication in the middle ear and thus bacterial OM. Following sialic acid-mediated viral attachment, the $\mathrm{H} 3$ HA may promote more effective interactions with secondary receptors required for virus entry and replication [14]. Alternatively, H3 HAs may display increased membrane fusion with epithelial cells in the middle ear, thereby promoting more efficient viral replication [15]. Thus, whilst $\mathrm{OM}$ is independent of tropism for $\alpha 2,6$-linked sialic acid, the other features of the HA which may mediate secondary bacterial disease remain an area of ongoing research.

\section{Additional file}

Additional file 1: Figure S1. Sialic acid expression in the murine middle ear following infection with Streptococcus pneumoniae (Sp) and/or the IAV strain Udorn/72. Sections were labeled with Maackia amurensis agglutinin (MAA; a2,3-linked sialic acid), Sambucus nigra agglutinin (SNA; a2,6-linked sialic acid) or unlabelled. Positive staining is shown by an arrow. MEE: Middle Ear Epithelium; MEC: Middle Ear Cavity.

\section{Abbreviations}

CFU: Colony forming units; HA: Hemagglutinin; $\mathrm{HS}^{\mathrm{R}}$ : Horse serum resistant; HMEECs: Human middle ear epithelial cells; IAV: Influenza A virus;

i.n: intranasally; OM: Otitis media; PFU: Plaque forming units.
Competing interests

The authors declare that they have no competing interests.

\section{Authors' contributions}

KRS: Performed experiments, assisted in study design and wrote the manuscript; MNH \& JP: Performed experiments, study design and data analysis; PCR/OLW: Assisted in study design \& editing manuscript DAD: Assisted in study design and data analysis, performed experiments and helped draft manuscript. All authors read and approved the final manuscript.

\section{Acknowledgments}

We wish to thank Ms. Kathryn Edenborough and Ms. Emma Job for their experimental assistance. KRS is supported by a GlaxoSmithKline post-graduate support grant and the Elizabeth and Vernon Puzey post-graduate research scholarship. DAD is supported by the 7th Framework Programme of the European Commission (ETB grant 08010). OLW is supported by a Career Development Fellowship (RD Wright Fellowship) from the Australian National Health and Medical Research Council and Pfizer-funded Robert Austrian Award. The Melbourne WHO Collaborating Centre for Reference and Research on Influenza is supported by the Australian Government Department of Health and Ageing. The funding sources of this work did not influence the design or analysis of the data presented in this manuscript.

\section{Author details}

'Department of Microbiology and Immunology, The University of Melbourne, Melbourne, VIC 3010, Australia. 'Laboratory of Pediatric Infectious Diseases, Department of Pediatrics, Radboud University Medical Centre, Nijmegen, The Netherlands. ${ }^{3}$ Australian Animal Health Laboratory, Geelong, VIC 3220, Australia. ${ }^{4} \mathrm{WHO}$ Collaborating Centre for Reference and Research on Influenza, Victorian Infectious Diseases Reference Laboratory, Melbourne, VIC 3051, Australia.

Received: 30 November 2012 Accepted: 15 April 2013

Published: 23 April 2013

\section{References}

1. Massa HM, Cripps AW, Lehmann D: Otitis media: viruses, bacteria, biofilms and vaccines. Med J Aus 2010, 191:S44-S49.

2. Peltola VT, Boyd KL, McAuley JL, Rehg JE, McCullers JA: Bacterial sinusitis and otitis media following influenza virus infection in ferrets. Infect Immun 2006, 74:2562-2567.

3. Giebink GS, Berzins IK, Marker SC, Schiffman G: Experimental otitis media after nasal inoculation of streptococcus pneumoniae and influenza a virus in chinchillas. Infect Immun 1980, 30:445-450.

4. Short KR, Diavatopoulos DA, Thorton $R$, et al: Influenza virus induces bacterial and non-bacterial otitis media. J Infect Dis 2011, 204:1857-1865.

5. Short KR, Reading PC, Brown LE, et al: Influenza-induced inflammation drives pneumococcal otitis media. Infect Immun 2012, 81(3):645-652.

6. Connor R, Kawaoka Y, Webster R, Paulson J: Receptor specificity in human, avian, and equine $\mathrm{H} 2$ and $\mathrm{H} 3$ influenza virus isolates. Virol 1994, 205:17-23.

7. Shinya K, Ebina M, Yamada S, Ono M, Kasai N, Kawaoka Y: Avian flu: influenza virus receptors in the human airway. Nature 2006, 440:435-436.

8. Ryan-Poirier K, Suzuki Y, Bean WJ, et al: Changes in H3 influenza A virus receptor specificity during replication in humans. Virus Res 1998, 56:169-176.

9. Tate MD, Brooks AG, Reading PC: Correlation between sialic acid expression and infection of murine macrophages by different strains of influenza virus. Microbes Infect 2011, 13:202-207.

10. Ibricevic A, Pekosz A, Walter MJ, et al: Influenza virus receptor specificity and cell tropism in mouse and human airway epithelial cells. J Virol 2006, 80:7469-7480

11. Diavatopoulos D, Short K, Price J, et al: Influenza A virus facilitates streptococcus pneumoniae transmission and disease. FASEB J 2010, 24:1789-1798.

12. Chun YM, Moon SK, Lee HY, et al: Immortalization of normal adult human middle ear epithelial cells using a retrovirus containing the E6/E7 genes of human papillomavirus type 16. Ann Otol Rhinol Laryngol 2002, 111:507-517. 
13. Anders E, Scalzo A, Rogers G, White D: Relationship between mitogenic activity of influenza viruses and the receptor-binding specificity of their hemagglutinin molecules. J Virol 1986, 60:476-482.

14. Chu VC, Whittaker GR: Influenza virus entry and infection require host cell N-linked glycoprotein. PNAS 2004, 101:18153-18158.

15. Koerner I, Matrosovich MN, Haller O, Staeheli P, Kochs G: Altered receptor specificity and fusion activity of the hemagglutinin contribute to high virulence of a mouse-adapted influenza A virus. J Gen Virol 2012, 93:970-979.

doi:10.1186/1743-422X-10-128

Cite this article as: Short et al: Influenza A virus induced bacterial otitis media is independent of virus tropism for a2,6-linked sialic acid. Virology Journal 2013 10:128.

\section{Submit your next manuscript to BioMed Central and take full advantage of:}

- Convenient online submission

- Thorough peer review

- No space constraints or color figure charges

- Immediate publication on acceptance

- Inclusion in PubMed, CAS, Scopus and Google Scholar

- Research which is freely available for redistribution 